\title{
Ruolo del rene nell'equilibrio acido-base
}

\author{
C. Queirolo, P. Solari, G. Delfino \\ Unità Operativa di Nefrologia Dialisi \\ Ospedali Riuniti del Tigullio \\ USL IV, Lavagna - Regione Liguria
}

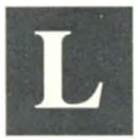

a concentrazione idrogenionica $\left[\mathrm{H}^{+}\right]$è una delle costanti che l'organismo tende a mantenere fissa con minime oscillazioni perché ogni deragliamento è incompatibile con la vita. Giornalmente dal metabolismo intermedio vengono prodotti $50-100 \mathrm{mEq}$ di $\mathrm{H}^{+}$ (0.3-1 mEq/kg/p.c.) immediatamente tamponati da sistemi all'uopo preposti. In prima linea operano i cosiddetti "sistemi tampone"(1) ma poiché questi non sono inesauribili, è indispensabile una costante e continua reintegrazione. L'organismo dispone di strutture in grado di funzionare, da un lato, come reintegratori del patrimonio dei sistemi tampone, dall'altro come veri emuntori in grado di allontanare definitivamente gli $\mathrm{H}^{+}$prodotti. L'organo preposto a questi compiti è il rene, in grado di intervenire con due meccanismi tra loro strettamente interdipendenti:

$1^{\circ}$ - Riassorbimento dei bicarbonti $\left(\mathrm{HCO}_{3}^{-}\right)$filtrati.

$2^{\circ}$ - Escrezione di $\mathrm{H}^{+}$sotto forma di " $\mathrm{H}^{+}$ liberi", acidità titolabile (AT), produzione di ione ammonio $\left(\mathrm{NH}_{4}^{+}\right)$, in quantità sufficiente da rigenerare gli $\mathrm{HCO}_{3}{ }^{-}$consumati per tamponare gli $\mathrm{H}^{+}$prodotti.

\section{Riassorbimento dei bicarbonati}

Un rene normale produce non meno di $100 \mathrm{ml} / \mathrm{min}$ di ultrafiltrato nella composizione del quale la concentrazione degli $\mathrm{HCO}_{3}{ }^{-}$è circa $25 \mathrm{mEq} / \mathrm{L}$.

Se rapportiamo il calcolo alle 24 ore la quantità di $\mathrm{HCO}_{3}{ }^{-}$filtrata supera di circa 5 volte il patrimonio dell'organismo; Poiché non è possibile una tale perdita, il rene deve provvedere al recupero degli $\mathrm{HCO}_{3}{ }^{-}$filtrati.

Circa il $90 \%$ della quota di $\mathrm{HCO}_{3}{ }^{-}$filtratta viene riassorbito in sede prossimale mediante una serie di reazioni tra loro strettamente collegate. Nella cellula tubulare la $\mathrm{CO}_{2}$ è presente in forma disciolta in equilibrio con $1^{\prime} \mathrm{H}_{2} \mathrm{O}$ con conseguente formazione di $\mathrm{H}_{2} \mathrm{CO}_{3}$.

La reazione è fortemente accelerata dall'anidrasi carbonica (AC) (2):

$$
\mathrm{CO}_{2}+\mathrm{H}_{2} \mathrm{O} \stackrel{\mathrm{AC}}{\longrightarrow} \mathrm{H}_{2} \mathrm{CO}_{3}
$$

Come tutti gli acidi, l'acido carbonico in soluzione acquosa si dissocia liberando idrogenioni $\left(\mathrm{H}^{+}\right)$ed anioni $\left(\mathrm{A}^{-}\right)$:

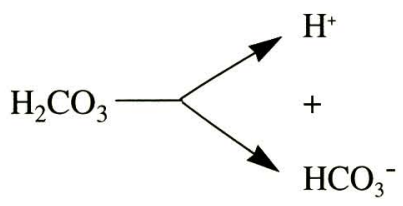

Mentre gli $\mathrm{H}^{+}$vengono attivamente trasportati (pompa ionica ATPasi-dipendente) nel lume tubulare, gli $\mathrm{HCO}_{3}^{-}$rimangono nella cellula (Fig. 1). La fuoriuscita degli $\mathrm{H}^{+}$crea un'asimmetria elettrica ai due lati della membrana cellulare responsabile del potenziale elettrico transmembranale.

Per la legge della neutralità elettrica, la fuoriuscita degli $\mathrm{H}^{+}$deve essere accompaganta da un'equivalente quantità di cariche negative orientate nella stessa direzione, o sostituita da altrettante cariche positive orientate in direzione opposta. Poiché la membrana cellulare dal lato luminale è impermeabile agli $\mathrm{HCO}_{3}{ }^{-}$, sono gli ioni sodio $\left(\mathrm{Na}^{+}\right)$, filtrati dal glomeru- 


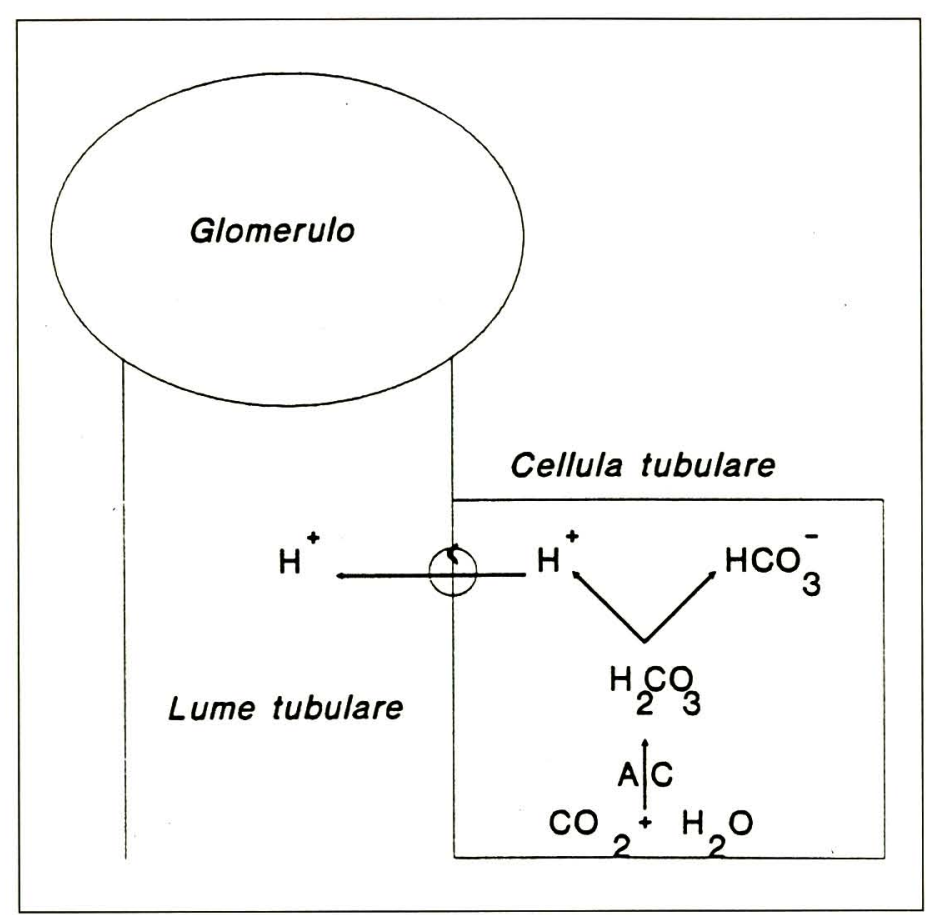

Fig. 1 - Produzione endocellulare e trasporto endoluminale del $\mathrm{H}^{+}$.

lo e presenti nel fluido luminale a sostituire gli $\mathrm{H}^{+}$(3).

Il $\mathrm{Na}^{+}$nell'ultrafiltrato si accompagna agli $\mathrm{HCO}_{3}^{-}$, quando viene sostituito dagli $\mathrm{H}^{+}$si ha la formazione di acido carbonico (Fig. 2).

L'AC che opera anche a livello luminale, provvede ad una rapida disidratazione dell'acido con formazione di $\mathrm{CO}_{2}$ ed $\mathrm{H}_{2} \mathrm{O}$ :

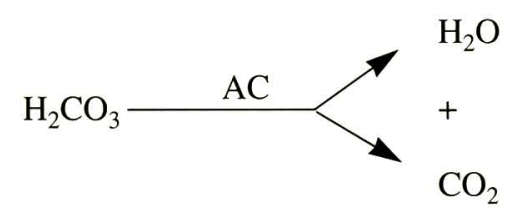

In conclusione $1^{\prime} \mathrm{HCO}_{3}{ }^{-}$filtrato viene dissipato in $\mathrm{H}_{2} \mathrm{O}$ che è un prodotto neutro ed in $\mathrm{CO}_{2}$ che come tutti i gas, diffonde liberamente in tutte le direzioni ad una velocità che è una funzione diretta della pressione parziale $\left(\mathrm{pCO}_{2}\right)$. $\mathrm{La}$ quota di $\mathrm{CO}_{2}$ che supera la cellula rientra nel pool corporeo e come tale viene eliminata con la respirazione (Fig. 3). complesso. È stata dimostrata la presenza di un controtrasporto $\mathrm{Na}^{+} / \mathrm{K}^{+}$la cui composizione deve essere ancora verificata. me.

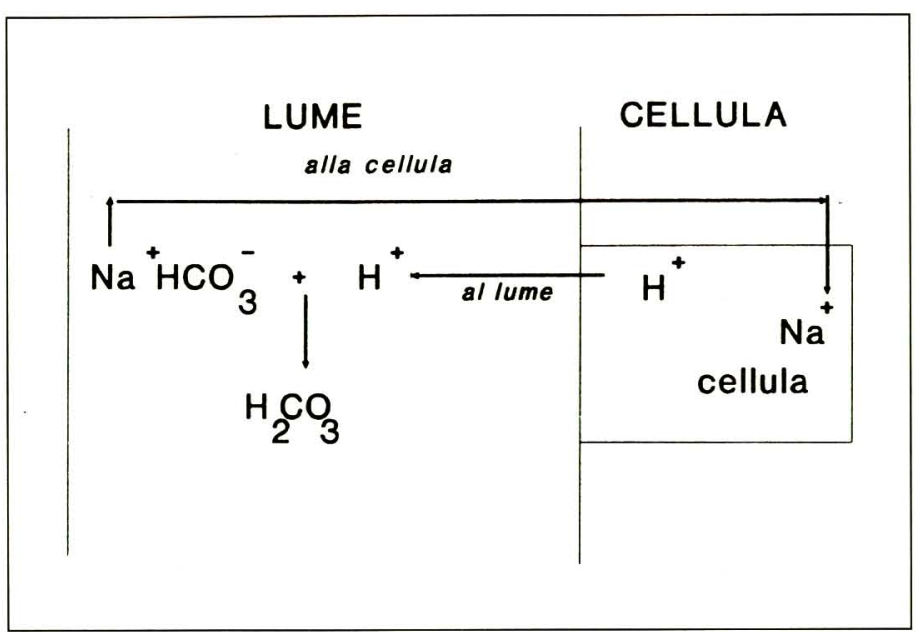

Fig. 2

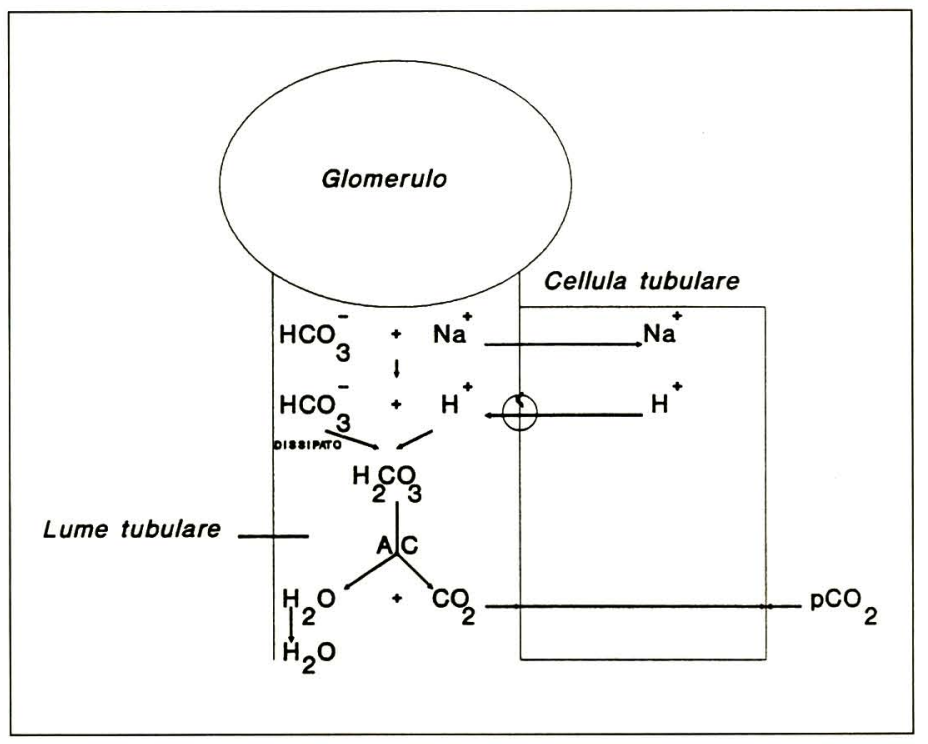

Fig. 3 - Scambi $\mathrm{H}+\mathrm{Na}^{+}$ tra cellula tubulare e lu-

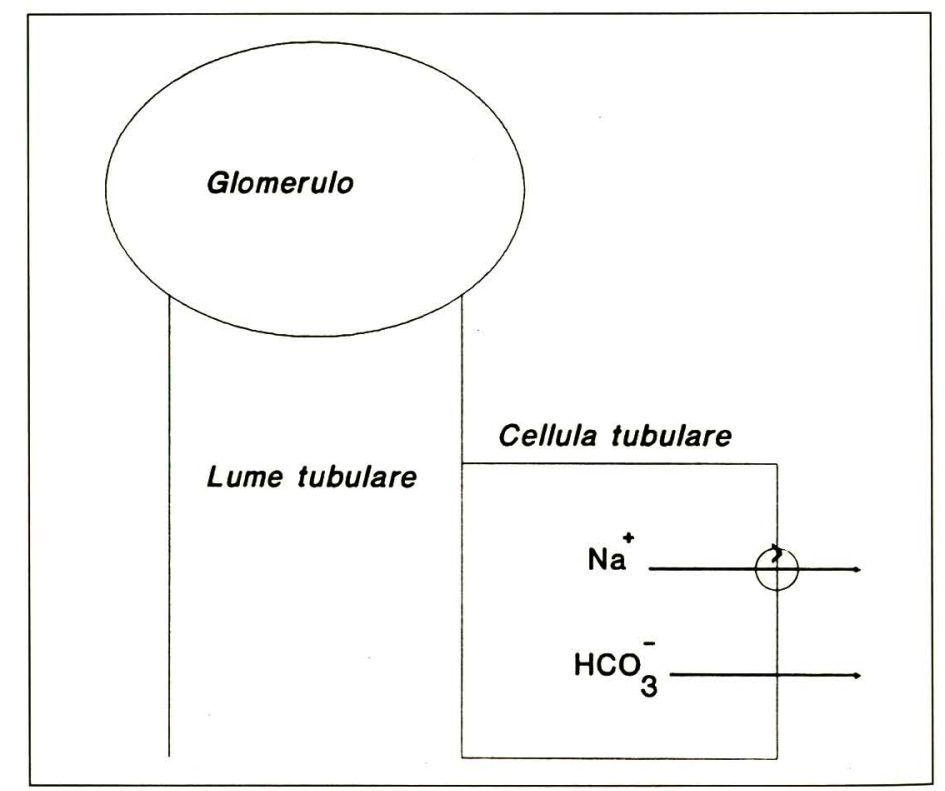

Fig. 4 - Recupero degli $\mathrm{HCO}_{3}{ }^{-}$ 


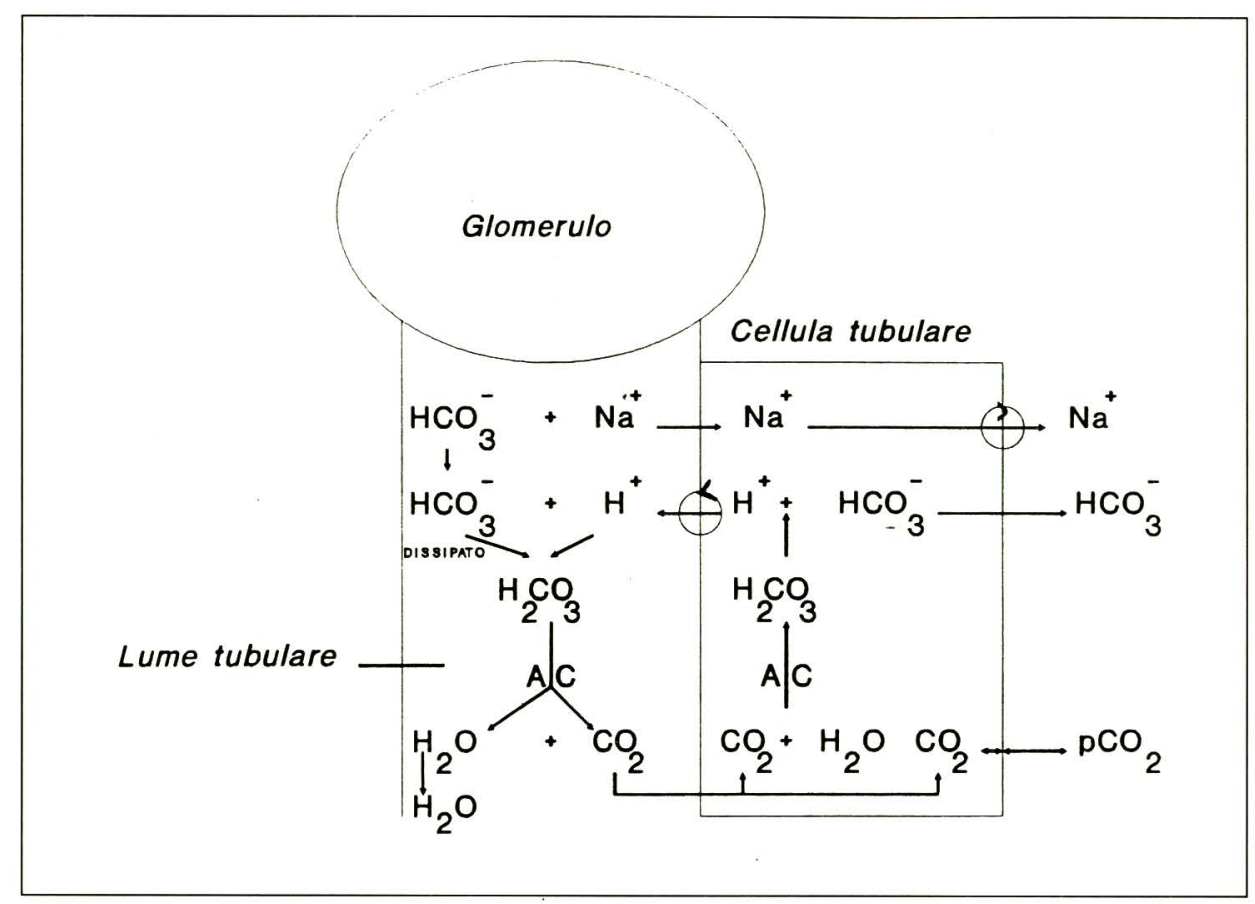

Fig. 5 - Produzione dei bicarbonati in sede prossimale.

Parallelamente a quanto accade sul versante luminale, in sede cellulare si svolgono altre reazioni. La membrana, dal lato antiluminale, è dotata di un sistema in grado di estromettere il $\mathrm{Na}^{+}$il quale è seguito dall' $\mathrm{HCO}_{3}{ }^{-}$con totale recupero dell'anione (Fig. 4). In realtà mentre lo ione $\mathrm{Na}^{+}$che rientra nel circolo è lo stesso che è stato filtrato, $\mathrm{l}^{\prime} \mathrm{HCO}_{3}^{-}$filtrato viene totalmente dissipato; quello che rientra è un $\mathrm{HCO}_{3}^{-}$" diverso", ma questo non cambia la sostanza delle cose (Fig. 5).

Se consideriamo l'estrema semplicità del sistema ed il fatto che non può mai essere saturo perché i prodotti della reazione $\left(\mathrm{H}_{2} \mathrm{O}\right.$ e $\left.\mathrm{CO}_{2}\right)$ vengono rapidamente allontanati, riesce difficile spiegare per quale ragione il segmento prossimale non sia in grado di provvedere al totale riassorbimento degli $\mathrm{HCO}_{3}^{-}$filtrati. Può essere chiamata in causa un'insufficiente produzione e/o secrezione idrogenionica $\mathrm{o}$, in alternativa, l'entrata in funzione di un meccanismo in grado di stoppare il processo stesso che, dal punto di vista chimico, potrebbe essere di entità ben superiore. Nel percorrere il segmento prossimale, l'ultrafiltrato affianca alla perdita di $\mathrm{HCO}_{3}{ }^{-}$, una perdita isotonica di $\mathrm{H}_{2} \mathrm{O}$ ed altri ioni $\left(\mathrm{Na}^{+}, \mathrm{Cl}^{-}\right.$, ecc. $)$ed una progressiva caduta del $\mathrm{pH}$; ciò crea un gradiente tra lume tubulare e cellula. Il trasporto degli $\mathrm{H}^{+}$avviene contro que- sto gradiente che rappresenta la forza in grado di opporsi al trasporto e può anche bloccarlo.

Il sistema di screzione idrogenionica, altamente efficiente in termini quantitativi, è, nel contempo, dotato di scarsa potenza, ossia può operare solo contro piccoli gradienti. Pertanto potrebbe essere lo stesso trasporto ad attivare le forze che ne limitano l'efficienza. L'aumento della quota di $\mathrm{HCO}_{3}^{-}$filtrato, limitando la caduta del $\mathrm{pH}$, aumenta il trasporto stesso, il quale tuttavia rimane indipendente dalla quota di $\mathrm{HCO}_{3}^{-}$filtrata ed è regolato esclusivamente dal $\mathrm{pH}$ (4).

Studi sperimentali su animali integri, su segmenti di tubulo isolato e su volontari, hanno dimostrato che il riassorbimento degli $\mathrm{HCO}_{3}^{-}$aumenta parallelamente al1'aumento della quota filtrata sino a un certo punto. È il cosiddetto $\mathrm{TmHCO}_{3}{ }^{-}$ (5), oltre il quale tutto $1^{\prime} \mathrm{HCO}_{3}^{-}$filtrato viene perduto (6).

In conclusione il tubulo prossimale è in grado di riassorbire la maggiore frazione di $\mathrm{HCO}_{3}{ }^{-}$filtrata riesce a mantenere concentrazioni leggermente superiori ai livelli massimi normali, riesce a impedire perdite di $\mathrm{HCO}_{3}{ }^{-}$per valori fisiologici della loro concentrazione anche se, molto probabilmente, la percentuale della quota filtrata che viene riassorbita decresce proporzionalmente alla quantità as- soluta che viene riassorbita. In presenza di disordini dell'equilibrio acido base non esiste limite all'escrezione idrogenionica ed al riassorbimento di $\mathrm{HCO}_{3}{ }^{-}$ anche se questo, nel segmento prossimale, rimane incompleto.

\section{Ruolo del segmento tubulare distale}

Sebbene solo il $10 \%$ circa della quota di $\mathrm{HCO}_{3}{ }^{-}$filtrata sfugga al riassorbimento prossimale, se non intervenissero i segmenti a valle, la perdita giornaliera raggiungerebbe i $324 \mathrm{mEq}$, quota che corrisponde al $50 \%$ delle riserve di basi dell'organismo; I due segmenti tubulari hanno obiettivi comuni anche se vengono raggiunti con differenti modalità. Il segmento prossimale ha elevata capacità e notevole flessibilità, ma è scarsamente modulabile. Al contrario il tubulo distale accoppia ad una scarsa capacità, una elevata sensibilità, una estrema manegevolezza ed una notevole adattabilità funzionale.

Il meccanismo con il quale le cellule epiteliali del segmento distale producono $\mathrm{H}^{+}$è identico a quanto visto a monte, inoltre sono in grado di secernere $\mathrm{H}^{+}$ contro elevati gradienti se si considera che il pH urinario può scendere a valori di 4.4 .

Se consideriamo che a pH 5 tutti gli $\mathrm{HCO}_{3}{ }^{-}$sono scomparsi dal fluido luminale, si deve ammettere che la secrezione idrogenionica prosegue anche in assenza di accettori. Sono i cosiddetti " $\mathrm{H}^{+}$liberi" ed il processo viene stoppato quando la $\left[\mathrm{H}^{+}\right]$urinaria supera di circa 1.000 volte la concentrazione plasmatica.

La differenza tra le due concentrazioni indica la massima energia che la pompa riesce a produrre per superare il gradiente di concentrazione, ma non spiega l'intimo meccanismo che permette alla secrezione idrogenionica di proseguire

(4) a valori di $\mathrm{pH}$ a 6.8 tutti gli $\mathrm{HCO}_{3}{ }^{-}$sono riassorbiti.

(5) $\mathrm{TmHCO}_{3}^{-}=$trasporto tubulare massimo. (6) eleganti studi sperimentali hanno dimostrato che, se durante l' infusione di $\mathrm{HCO}_{3}{ }^{-}$ s'impedisce l'espansione dell' extracellulare, il $\mathrm{TmHCO}_{3}{ }^{-}$non viene mai raggiunto. 
anche quando si è completato il riassorbimento degli $\mathrm{HCO}_{3}{ }^{-}$. Se si considera la discrepanza tra $\mathrm{pH}$ urinario e $\mathrm{pH}$ plasmatico si può sospettare che gli $\mathrm{H}^{+}$ vengano secreti come tali.

Considerando che per valori di pH urinario di 4 (7) l'escrezione massima di $\mathrm{H}^{+}$liberi sarebbe di circa $0.1 \mathrm{mEq} / \mathrm{L}$ di urina, è evidente l'insufficienza di questa via di smaltimento rispetto alla produzione. Gli $\mathrm{H}^{+}$, per essere eliminati, devono essere coniugati e veicolati con basi che fungano da accettori.

Gli acidi forti contenuti negli alimenti in forma neutra vengono liberati dal metabolismo ossidativo ma non circolano come tali, essi reagiscono con un equivalente quantitativo di $\mathrm{HCO}_{3}^{-}$trasformandosi nei corrispondenti sali neutri (Figg. 6, 7).

Nel plasma e quindi nell'ultrafiltrato il rapporto fosfato bisodico/fosfato monosodico è di 4/1. Questi sali sono facilmente filtrabili, ma difficilmente riassorbibili. Nel lume vengono titolati dagli $\mathrm{H}^{+}$ prodotti dalla cellula, ritrasformati in acidi e poiché il grado di dissociazione dipende dal $\mathrm{pK}$ dell'acido stesso, gran parte degli $\mathrm{H}^{+}$rimangono confinati nella molecola ed eliminati.

\section{Produzione ed eliminazione dell'acidità titolabile}

Gli anioni fosfato, solfato, ecc. sono gli stessi che hanno liberato gli $\mathrm{H}^{+}$metabolici e vengono titolati dagli stessi idrogenioni. La reazione, in vivo, non è stechiometrica; il $\mathrm{pH}$ urinario non scende a valori così bassi da permettere l'eliminazione di acido fosforico, solforico, ecc. Questi acidi vengono eliminati rispettivamente come $\mathrm{NaH}_{2} \mathrm{PO}_{4}$ e $\mathrm{NaHSO}_{4}$, quindi ogni $5 \mathrm{H}^{+}$prodotti, 2 vengono trattenuti, quindi non tutti gli $\mathrm{HCO}_{3}{ }^{-}$dissipati all'inizio della reazione vengono rigenerati.

Questo tipo di acidità viene definita "Titolabile" perché la sua entità può es-

(7) la massima acidità raggiungibile dell' urina ha $\mathrm{pH}=4.4$ sere facilmente valutata in vitro riproducendo in provetta le stesse reazioni, in-

vertite nella direzione, che avvengono a livello del tubulo.

in vitro
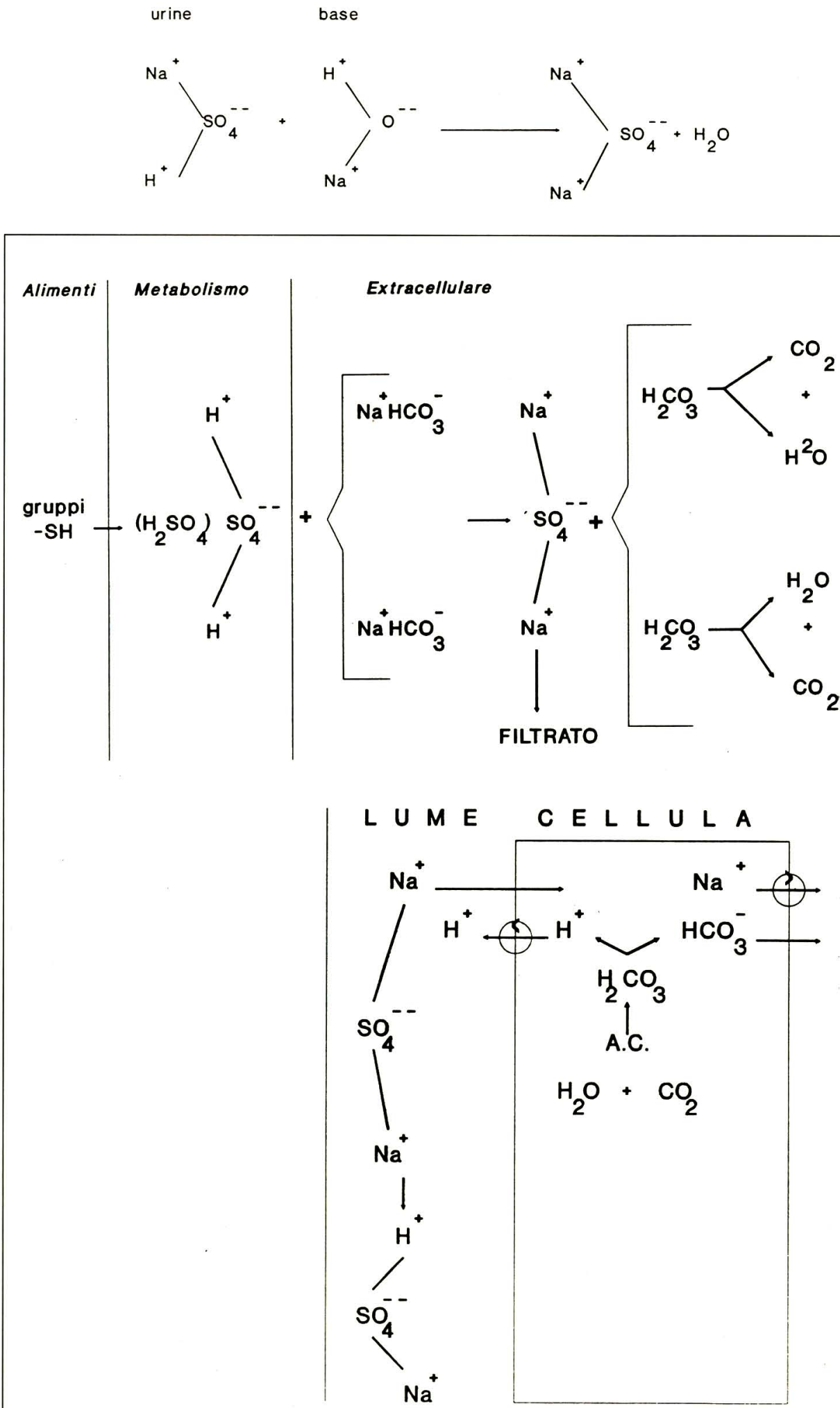


\section{Produzione di ammoniaca}

$\mathrm{L}^{\prime} \mathrm{HCO}_{3}{ }^{-}$che origina dalla dissociazione intracellulare dell' $\mathrm{H}_{2} \mathrm{CO}_{3}$, può essere recuperato dall'organismo se viene legato ad una base che deve necessariamente provenire dal lume (scambio $\mathrm{H}^{+} \longrightarrow \mathrm{Na}^{+}$) perché quelle confinate nella cellula sono quantitativamente estremamente scarse e/o comunque lega- te a gruppi anionici che dovrebbero essere metabolizzati a gruppi neutri prima che la base possa essere utilizzata a questo livello. Le basi, (rappresentate in prevalenza dal $\mathrm{Na}^{+}$) non sono più presenti nel lume perché tutte scambiate. Rimane il sodio legato al cloro sotto forma di sale neutro $(\mathrm{NaCl})$. Se l'accettore dell' $\mathrm{H}^{+}$ fosse il cloro, come avviene a livello gastrico, vi sarebbe recupero di $\mathrm{HCO}_{3}{ }^{-} \mathrm{e}$ produzione di $\mathrm{HCl}$. L'acido cloridrico è un acido forte, altamente dissociato con

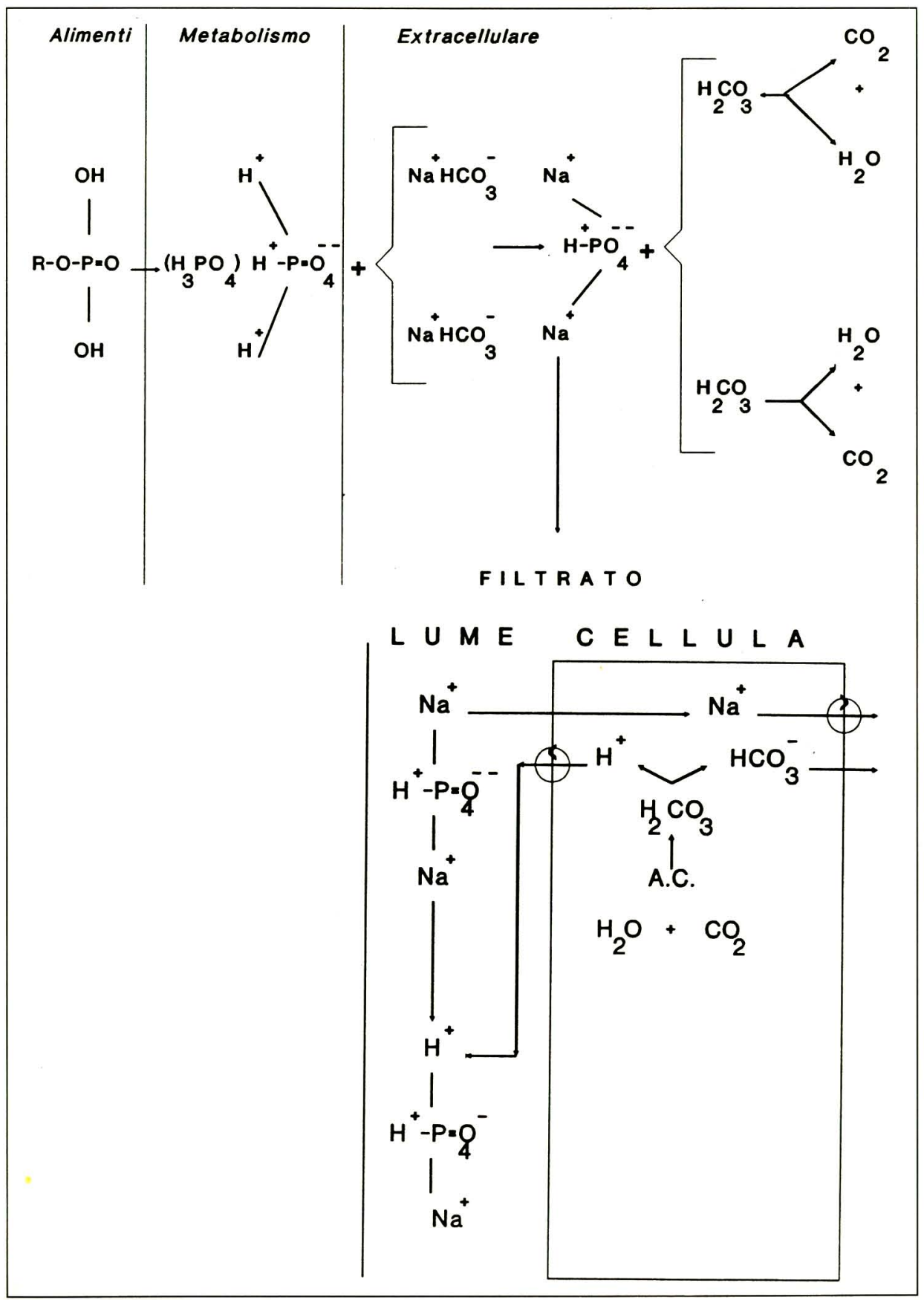

Fig. 7

una $\left[\mathrm{H}^{+}\right]$che porterebbe il $\mathrm{pH}$ urinario a valori nettamente inferiori a 4.4 (massima acidità delle urine). Poiché questa via non è percorribile, l'organismo deve sintetizzare una base per scambiarla con il $\mathrm{Na}^{+}$contenuta nel sale neutro. Specifici sistemi enzimatici, particolarmente attivi in corso di acidosi metabolica, facilitano la captazione di diversi aminoacidi, in particolare della glutamina, da parte delle cellule tubulari (segmento prossimale e distale). La glutamina viene metabolizzata ad acido alfa chetoglutarico (8) e a 2 molecole di $\mathrm{NH}_{3}$. L' $\mathrm{NH}_{3}$ è un gas che in soluzione acquosa presenta le stesse caratteristiche degli idrossidi alcalini, ossia si comporta come una base forte:

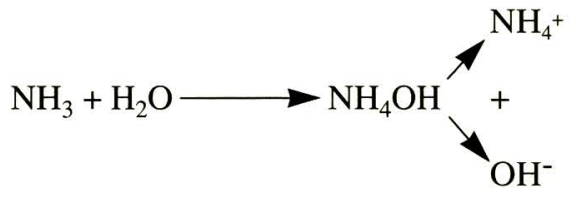

A livello cellulare si realizza un equilibrio tra ammoniaca e suo radicale acido regolato dal $\mathrm{pH}$ intracellulare:

$\mathrm{NH}_{3}+\mathrm{H}^{+} \longrightarrow \mathrm{NH}_{4}^{+}$

Per valori di $\mathrm{pH}$ intracellulare di 7.30 il rapporto $\mathrm{NH}_{3} / \mathrm{NH}_{4}{ }^{+}$è di circa $1 / 100 \mathrm{e}$ l' $\mathrm{NH}_{3}$ che diffonde fuori dalla cellula viene immediatamente ricostituito dai depositi di ammonio. Il gas ammoniaca è un gas ed esercita una pressione parziale $\left(\mathrm{pNH}_{3}\right)$ che è tanto maggiore quanto minore è la $\left[\mathrm{H}^{+}\right]$intracellulare. Poiché gli $\mathrm{H}^{+}$prodotti dalla cellula vengono liberati nel lume dove possono raggiungere concentrazioni circa 1.000 volte superiori, la $\mathrm{pNH}_{3}$ saré enormemente inferiore in sede luminale e $1^{\prime} \mathrm{NH}_{3}$ potrà defluire dal luogo di produzione al lume del tubulo. L'elevata concentrazione idrogenionica,

(8) l'acido alfachetoglutarico o viene immesso nel ciclo di Krebs ed ossidato a $\mathrm{CO}_{2}$ e $\mathrm{H}_{2} \mathrm{O}$ oppure convertito in fosfo-enolpiruvico $e$ successivamente in glucosio. Entrambe le vie sintetizzano prodotti neutri e la reazione non si stoppa. 


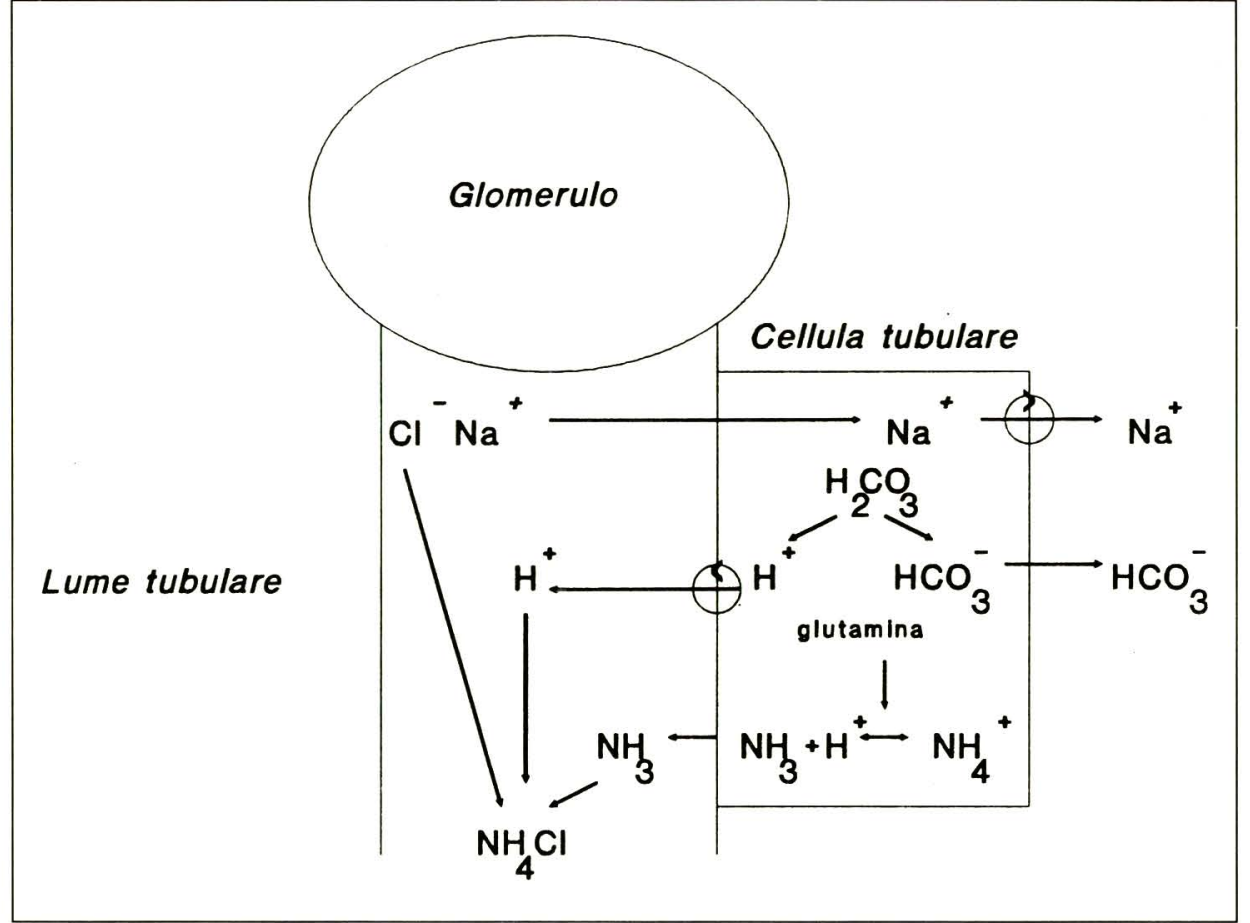

Fig. 8 - Produzione di ammoniaca

ossia il basso $\mathrm{pH}$ urinario (9) spingono alla formazione di $\mathrm{NH}_{4}^{+}$ione non gassoso, idrosolubile, non diffusibile. Nel lume l'accumulo di $\mathrm{NH}_{4}{ }^{+}$stopperebbe la reazione se i cationi non venissero neutralizzati dal $\mathrm{Cl}^{-}$fornito dal sale neutro e reso disponibile dallo scambio $\mathrm{Na}^{+} \longrightarrow \mathrm{H}^{+}$. Il sale neutro prodotto $\left(\mathrm{NH}_{4} \mathrm{Cl}\right)$ contiene nella sua molecola un idrogenione che viene definitivamente allontanato (Fig. 8).

(9) la coppia $\mathrm{NH}_{3} / \mathrm{NH}_{4}^{+}$ha un $\mathrm{pK}=9.30$, quindi ad un $\mathrm{pH}$ di 9.30 l'ammoniaca sarebbe al $50 \%$ sotto forma di $\mathrm{NH}_{3}$ ed al $50 \%$ sotto forma di $\mathrm{NH}_{4}^{+}$. Poiché l'urina ha sempre un $\mathrm{pH}$ più basso, la coppia è presente esclusivamente in forma ionizzata $\left(\mathrm{NH}_{4}^{+}\right)$.

\section{BIBLIOGRAFIA}

1. Bartoli E. La medicina Internazionale n²6 Giugno 1979.

2. Brenner BM, Rector FC Jr. The Kidney W.B. Philadelphia: Saunders Company 1991.

3. Borgia MC, Luciani C, Speciale G. I Disturbi Connessi al Contenuto Corporeo di Acqua, Sodio e Potassio. Torino: Stampa Medica Febbraio 1990.

4. Collins DR. Illustrated Manual of Fluid and Electrolyte Disorders. Philadelphia: J.B. Lippincott Company 1976.

5. Davenport H.W. The ABC of acid-base chemistry. University of Chicago 1969.

6. Garella S, Gallieni M. Problem Solving. Milano:Wichtig Editore 1989.

7. Goldeberger E. A Primer of water, electrolyte and acid-base syndromes. S.E.U. 1974.
8. Grassi V, Sorbini CA. L'Equilibrio acido-base in medicina. Medicina illustrata n. 15 anno IV Settembre. Il Pensiero Scientifico 1973.

9. Maschio G. Il metabolismo elettrolitico e minerale nelle malattie renali. Padova: Piccin Edit. 1980.

10. Minetti L, Colussi G, De Ferrari ME, Rombola G. Esplorazione Funzionale del Tubulo Renale. Milano: Wichtig Editore 1992.

11. Ole Siggaard-Andersen. The Acid-Base Status of the Blood MunKsgaard-international. Copenhagen: Booksellers \& Publishers Limited 1971.

12. Petrella E. Le basi fisiopatologiche degli squilibri idroelettrolitici e acido-base. McGraw-Hill Libri Italia 1990.

13. Rose D.B. Fisiologia clinica dell'equilibrio acido-base e dei disordini elettrolitici. McGraw-Hill Libri Italia 1991. 